\section{Fatores associados à continuidade interpessoal na atenção à saúde: estudo de base populacional}

\author{
Factors associated with interpersonal continuity of \\ care: a population-based study
}

\author{
${ }_{1}^{1}$ Programa de Pós-graduação \\ em Epidemiologia, \\ Universidade Federal de \\ Pelotas, Pelotas, Brasil. \\ Correspondência \\ L. A. Rosa Filho \\ Programa de Pós-gradu- \\ ação em Epidemiologia \\ Universidade Federal de \\ Pelotas. \\ Av. Duque de Caxias 250, \\ 3 o andar, Pelotas, RS \\ 96030-002, Brasil. \\ lzartur@yahoo.com.br
}


e a responsabilização das equipes de saúde com suas populações.

Em 2006, o Ministério da Saúde brasileiro estabeleceu a política nacional da atenção básica, reafirmando a opção substitutiva do PSF como a estratégia de reorganização deste nível de atenção 11. Essa política é orientada pelos princípios do vínculo e continuidade, bem como integralidade, entendida como um conjunto articulado e contínuo das ações em todos os níveis de complexidade. Entretanto, muitos estudos identificaram dificuldades de implantação do PSF em cidades de médio/grande porte 12 .

Historicamente, a continuidade é um conceito de difícil definição e medida. A diversidade de indicadores, aparentemente não conectados, reflete fenômenos complementares da continuidade. Em 2003, os estudos de Saultz ${ }^{1}$ e Haggerty 13 propõem que a continuidade seja classificada em três categorias: (a) continuidade de informação, cuja característica principal é a disponibilidade das informações de saúde do paciente para o profissional que for prestar o cuidado; (b) continuidade longitudinal/gerencial, é aquela em que o paciente tem um local e uma equipe específica a fim, que realiza os cuidados em saúde; (c) continuidade interpessoal, refere-se a situações em que o paciente conhece seu médico pelo nome e confia neste. Ao mesmo tempo o profissional assume a responsabilidade pelo cuidado do paciente 1 .

O único estudo de base populacional brasileiro 14 mostrou que, no Sul do Brasil, em 1.260 indivíduos maiores e 15 anos, a prevalência de médico de referência foi de $37,4 \%$. Nesse estudo, a continuidade interpessoal na atenção à saúde esteve associada ao sexo feminino, aumento da idade, maiores rendas familiares per capita, plano de saúde privado, relato de doença crônica e autopercepção ruim quanto à saúde. Em 2004, um estudo transversal americano realizado com 60.446 pessoas, encontrou que a continuidade, definida pela confirmação de que consultam com o mesmo médico em seu lugar de referência, esteve associada ao sexo feminino, idades avançadas, cor branca, melhores níveis educacionais e não fumantes 15 .

Este estudo tem como objetivo avaliar a prevalência de continuidade interpessoal na atenção à saúde e seus determinantes sócio-econômicos, demográficos, assistenciais e relacionados às necessidades de saúde dos indivíduos. Essas informações poderão apoiar políticas de ampliação da continuidade interpessoal na atenção à saúde no Sistema Único de Saúde (SUS).

\section{Metodologia}

Foi realizado um estudo transversal de base populacional com os indivíduos de 20 anos ou mais, da zona urbana de Pelotas, Rio Grande do Sul, Brasil. A cidade está localizada no extremo sul do Brasil, tem 320 mil habitantes e possui boa infraestrutura de saúde, contando com 54 unidades básicas de saúde.

O trabalho de campo foi conduzido de outubro a dezembro de 2005 e foi realizado em conjunto por 11 mestrandos do Programa de Pósgraduação em Epidemiologia da Faculdade de Medicina, Universidade Federal de Pelotas.

Foram realizados cálculos de tamanho de amostra para contemplar o maior número de indivíduos necessários para estudar a prevalência de continuidade interpessoal na atenção à saúde e suas associações com as variáveis independentes estudadas. Para o estudo descritivo, se estimou uma prevalência de $37 \%$, com erro aceitável de $3 \%$, chegando-se a um tamanho de amostra de 992 indivíduos, que acrescidos de 10\% para perdas e recusas, totalizou 1.091 pessoas. Para o estudo das associações, com nível de significância de $95 \%$ e poder estatístico de $80 \%$, calculou-se um tamanho de amostra para freqüência de $34 \%$ em não expostos, com RR (risco relativo) de 1,5 e razão de exposição de 1:4 para nível sócio-econômico, totalizando 458 indivíduos, que acrescidos de $10 \%$ para perdas, $20 \%$ para fatores de confusão e $20 \%$ para efeitos de delineamento exigem uma amostra mínima de 725 indivíduos. Em função do tamanho de amostra requerido pelos outros mestrandos, a amostra total foi de 3.353, ampliando o poder estatístico do estudo.

O processo de amostragem ocorreu em múltiplos estágios, baseado no Censo Demográfico de 2000 realizado pelo Instituto Brasileiro de Geografia e Estatística (IBGE). Com base nessa informação, foram sorteados, de forma sistemática, 120 dos 404 setores censitários com probabilidade proporcional ao seu tamanho. Nesses setores, todos os domicílios foram identificados, sendo sorteados 1.597 domicílios elegíveis. Em seguida, sorteou-se um número aleatório variável, de acordo com o número de domicílios e outro número correspondente ao pulo para garantir a eqüiprobabilidade de ser sorteado o primeiro domicílio.

Os domicílios selecionados foram visitados pelos supervisores de campo para definir a população em estudo. Foram selecionadas 33 entrevistadoras do sexo feminino e com ensino médio completo, que trabalharam em regime de dedicação integral e foram treinadas por 40 horas. Foi utilizado um questionário padronizado, caracterizando aspectos sócio-demográficos e 
temas específicos de cada pesquisador. Foram consideradas inelegíveis para o estudo pessoas com doença física ou mental, que estivessem incapacitadas para responder ao questionário. Aqueles que, após três visitas da entrevistadora em horários e dias diferentes e uma visita do supervisor de campo não puderam ser contatados, foram classificados como perdas. O controle de qualidade foi realizado pelos supervisores, predominantemente por telefone, em $10 \%$ da amostra total e, o menor Kappa encontrado foi de 0,72 para variável escolaridade.

Para a entrada dos dados utilizou-se o programa EpiInfo 6.0 (Centers for Disease Control and Prevention, Atlanta, Estados Unidos) com dupla entrada. A análise dos dados foi realizada no Stata 9.0 (Stata Corp., College Station, Estados Unidos), utilizando-se para descrição o teste do qui-quadrado de Pearson e teste de tendência linear. Considerou-se que tinha continuidade interpessoal na atenção à saúde quem relatava ter um médico pessoal há mais de um ano e sabia seu nome.

Na análise ajustada utilizou-se regressão "para trás” de Poisson com variância robusta, por tratar-se de um desfecho binário, porém freqüente. $\mathrm{O}$ efeito de delineamento amostral foi considerado através do comando svy para amostras por conglomerados. Para controle de fatores de confusão, o efeito de cada variável foi controlado para todas as variáveis de mesmo nível ou superior que tivessem valor $\mathrm{p} \leq 0,2$.

A análise foi hierarquizada conforme modelo com dois níveis. No primeiro, foram incluídas as variáveis sócio-econômicas: renda familiar no último mês, em Reais, categorizada em salários mínimos (um salário mínimo $=\mathrm{R} \$ 300,00$ em outubro/2005); escolaridade em anos completos de estudo, categorizada em Ensino Fundamental, Médio e Superior; e nível sócio-econômico segundo os critérios da Associação Brasileira de Empresas de Pesquisa (A, B, C, D e E) 16. Ainda no primeiro nível, foram inclusas as variáveis demográficas: idade, em anos completos; sexo; e cor da pele auto-referida, categorizada em brancos e não-brancos.

No segundo nível, foram incluídas variáveis relacionadas às necessidades em saúde: autopercepção em saúde (excelente, muito boa, boa, regular ruim) e existência de doença crônica referida. Incluiu-se também uma variável sobre a utilização dos serviços de saúde (ter consultado no último ano), bem como, uma variável sobre o modelo assistencial (forma de remuneração das consultas).

Realizou-se também uma análise multivariada sobre os determinantes da continuidade interpessoal na atenção à saúde, restringindo a população em estudo aos usuários de unidades básicas de saúde. Para isso, o modelo de análise incluiu no nível proximal a variável sobre a exposição ao PSF e excluiu a variável forma de financiamento da consulta, sendo no restante igual ao anteriormente apresentado.

O projeto foi aprovado pela Comissão de Ética em Pesquisa, Universidade Federal de Pelotas. Para participar da pesquisa, os entrevistados assinaram um termo de consentimento, lido pelo entrevistador, no qual constavam seus direitos de não participar ou suspender sua participação a qualquer momento e ao sigilo de informações.

\section{Resultados}

Foram entrevistados 3.136 indivíduos em 1.507 domicílios. As perdas e recusas corresponderam a $6,5 \%$ durante o trabalho de campo. A prevalência de continuidade interpessoal na atenção à saúde foi de 43,7\% (IC95\%: 42,0-45,5) com um efeito de delineamento amostral de 2,49 e correlação intraclasse de 0,05.

A Tabela 1 mostra que as mulheres constituíram $56,1 \%$ da amostra. A média de idade foi de 44 anos (IC95\%: 43,5-44,7), 54,4\% dos entrevistados estudaram oito anos ou menos e 81,6\% relataram cor da pele branca. Aproximadamente a metade da população tem renda familiar mensal de até três salários, havendo predomínio dos níveis sócio-econômicos B, C e D com 26,5\%,41,5\% e $24,5 \%$, respectivamente.

A maior parte $(73,2 \%)$ das pessoas consultou no último ano e $30 \%$ relataram a existência de doença crônica, mas $72 \%$ consideraram sua condição de saúde boa ou ótima. A forma de financiamento das consultas para $47,8 \%$ dos entrevistados foi o sistema público, $45,2 \%$ tinham plano de saúde e $7 \%$ referiram pagar por suas consultas médicas diretamente ao profissional (Tabela 1).

Quanto ao local de consulta, 33,6\% costumam consultar em unidades básicas de saúde, 25,9\% em consultórios médicos, 15 em Pronto-atendimento de convênio, 9\% em ambulatórios públicos, 5,5\% em pronto-socorro, 5,4\% em ambulatórios de convênio e 3,8\% relataram não consultar em lugar algum (Tabela 2). Entre as 1.052 pessoas que consultaram em unidades básicas de saúde, 39,5\% utilizaram unidades básicas de saúde com PSF.

Na análise bruta, sexo feminino, aumento da idade, escolaridade, classe social, renda familiar, consultar no último ano, relato de doença crônica, financiamento privado e autopercepção ruim sobre a própria saúde estiveram associados com continuidade interpessoal na atenção à saúde. Cor da pele não esteve associada. As variáveis 
Tabela 1

Características da população estudada conforme variáveis demográficas, sócio-econômicas, assistenciais e de necessidades em saúde. Pelotas, Rio Grande do Sul, Brasil, 2006

\begin{tabular}{|c|c|c|c|c|}
\hline \multirow[t]{2}{*}{ Variável } & \multicolumn{2}{|c|}{$\begin{array}{l}\text { População total } \\
\qquad(\mathrm{N}=3.133)\end{array}$} & \multicolumn{2}{|c|}{$\begin{array}{l}\text { Usuários de unidades básicas de saúde } \\
\qquad(N=1.052)\end{array}$} \\
\hline & $\mathbf{n}$ & $\%$ & $n$ & $\%$ \\
\hline \multicolumn{5}{|l|}{ Sexo } \\
\hline Masculino & 1.376 & 43,9 & 401 & 38,1 \\
\hline Feminino & 1.757 & 56,1 & 651 & 61,9 \\
\hline \multicolumn{5}{|l|}{ Idade (anos) } \\
\hline $20-29$ & 746 & 23,8 & 268 & 25,5 \\
\hline $30-39$ & 610 & 19,5 & 242 & 23,0 \\
\hline $40-49$ & 661 & 21,1 & 242 & 23,0 \\
\hline $50-59$ & 520 & 16,6 & 149 & 14,2 \\
\hline $60 \mathrm{ou}+$ & 596 & 19,0 & 151 & 14,3 \\
\hline \multicolumn{5}{|c|}{ Escolaridade (anos) } \\
\hline $0-4$ & 603 & 20,4 & 293 & 30,4 \\
\hline $5-8$ & 1.003 & 34,0 & 431 & 44,7 \\
\hline $9-11$ & 824 & 27,9 & 210 & 21,8 \\
\hline $12 \mathrm{ou}+$ & 521 & 17,7 & 30 & 3,1 \\
\hline \multicolumn{5}{|c|}{ Renda familiar (salários mínimos) } \\
\hline$<1$ & 360 & 11,6 & 173 & 16,5 \\
\hline $1-3$ & 1.127 & 36,2 & 580 & 55,5 \\
\hline $3-6$ & 842 & 27,1 & 215 & 20,5 \\
\hline $6 \mathrm{ou}+$ & 783 & 25,1 & 78 & 7,5 \\
\hline \multicolumn{5}{|c|}{ Classe social (ABEP) } \\
\hline$A$ & 161 & 5,2 & 4 & 0,4 \\
\hline B & 824 & 26,5 & 110 & 10,5 \\
\hline C & 1.293 & 41,5 & 474 & 45,3 \\
\hline $\mathrm{D}$ & 761 & 24,5 & 411 & 39,3 \\
\hline$E$ & 73 & 2,3 & 47 & 4,5 \\
\hline \multicolumn{5}{|l|}{ Cor da pele } \\
\hline Branca & 2.556 & 81,6 & 765 & 73,3 \\
\hline Não-branca & 577 & 18,4 & 279 & 26,7 \\
\hline \multicolumn{5}{|c|}{ Consulta no último ano } \\
\hline Não & 839 & 26,8 & 325 & 30,9 \\
\hline $\operatorname{Sim}$ & 2.294 & 73,2 & 727 & 69,1 \\
\hline \multicolumn{5}{|l|}{ Doença crônica } \\
\hline Não & 2.195 & 70,0 & 783 & 74,4 \\
\hline Sim & 938 & 30,0 & 269 & 25,6 \\
\hline \multicolumn{5}{|c|}{ Autopercepção de saúde } \\
\hline Excelente & 412 & 13,2 & 95 & 9,0 \\
\hline Muito boa & 418 & 13,3 & 94 & 8,9 \\
\hline Boa & 1.427 & 45,5 & 493 & 46,9 \\
\hline Regular & 722 & 23,0 & 306 & 29,1 \\
\hline Ruim & 154 & 5,0 & 64 & 6,1 \\
\hline \multicolumn{5}{|l|}{ Plano de saúde } \\
\hline Público & 1.499 & 47,8 & & \\
\hline Convênio & 1.416 & 45,2 & & \\
\hline Particular & 218 & 7,0 & & \\
\hline
\end{tabular}

ABEP: Associação Brasileira de Estudos Populacionais (http://www.abep.org/).

Nota: número máximo de perdas para a variável escolaridade (5,8\%). 
Características da população estudada conforme local de consulta. Pelotas, Rio Grande do Sul, Brasil, 2006 (N = 3.133).

\begin{tabular}{lcc}
\hline Local de consulta & População total & Distribuição \\
& $\mathbf{n}$ & $\%$ \\
\hline Unidade básica de saúde & 1.052 & 33,6 \\
Consultório médico & 812 & 25,9 \\
Pronto-atendimento/Convênio & 471 & 15,0 \\
Ambulatórios públicos & 283 & 9,0 \\
Pronto-socorro & 173 & 5,5 \\
Ambulatórios conveniados & 170 & 5,4 \\
Não consultou & 119 & 3,8 \\
Outros & 56 & 1,8 \\
\hline
\end{tabular}

escolaridade e classe social não foram incluídas no modelo por apresentarem co-linearidade com renda familiar, que foi a variável sócio-econômica com menor valor $\mathrm{p}$.

Na análise multivariável, após ajuste para fatores de confusão, o sexo feminino apresentou razão de prevalência (RP) de 1,66; IC95\%: 1,521,80. A idade apresentou associação linear direta com a continuidade interpessoal na atenção à saúde (valor $\mathrm{p}<0,001$ ), sendo que os adultos com mais de 60 anos tiveram $76 \%$ mais continuidade interpessoal na atenção à saúde do que aqueles entre 20 a 29 anos. A renda familiar apresentou associação direta com continuidade interpessoal na atenção à saúde, sendo que indivíduos com renda de seis ou mais salários tiveram $61 \%$; IC95\%: 1,39-1,87 mais continuidade interpessoal na atenção à saúde que aqueles que receberam até um salário (Tabela 3).

Ter consultado no último ano permaneceu associado significativamente à continuidade interpessoal na atenção à saúde com $\mathrm{RP}=1,47$; IC95\%: 1,29-1,67. A continuidade interpessoal na atenção à saúde foi significativamente mais prevalente naqueles que relataram doenças crônicas com $\mathrm{RP}=1,10$; IC95\%: 1,01-1,19. Ao analisarmos a forma de financiamento das consulta, os entrevistados que relataram ter plano de saúde ou pagar suas consultas por seus próprios meios tiveram mais continuidade interpessoal na atenção à saúde, com RP = 1,37; IC95\%: 1,24-1,52 e 1,64; 1,42-1,90, respectivamente.

Avaliou-se também os fatores associados à continuidade interpessoal na atenção à saúde entre as pessoas que consultaram no último ano, obtendo-se efeitos similares, com as mesmas variáveis compondo o modelo de regressão. Além disso, também repetiu-se a análise para aqueles que consultaram em unidades básicas de saú- de. A prevalência de continuidade interpessoal na atenção à saúde em unidades de PSF foi de $38,6 \%$, enquanto nas unidades básicas tradicionais foi de $31,8 \%$ (valor $\mathrm{p}=0,03$ ) (Tabela 4 ).

Entre aqueles que consultaram numa unidade básica de saúde, as mulheres tiveram duas vezes mais continuidade interpessoal na atenção à saúde do que os homens $(\mathrm{p}<0,001)$. Quando se estudou o efeito da idade sobre a continuidade interpessoal na atenção à saúde, houve tendência linear positiva, observou-se que indivíduos com mais de 60 anos tiveram RP = 1,97; IC95\%: 1,52-2,57, quando comparados com aqueles de 20 a 29 anos. Nessa população a renda não esteve associada à continuidade interpessoal na atenção à saúde, possivelmente pela sua homogeneidade, uma vez que nesta população há uma concentração de indivíduos com renda de até três salários mínimos (72\%). Ao analisarmos aqueles que consultaram em unidades básicas de saúde, consultar no último ano $(\mathrm{p}=0,01)$ foi a única das variáveis proximais associada à continuidade interpessoal na atenção à saúde, já que a autopercepção de saúde e o relato de doença crônica não estiveram associados.

\section{Discussão}

A prevalência de continuidade interpessoal na atenção à saúde encontrada na população foi consistente com o outro estudo brasileiro que utilizou o mesmo critério 14. Entretanto, considerando-se os indicadores de superutilização de serviços de saúde pela população, demonstrada em estudos anteriores 17,18, seria esperado uma maior prevalência de continuidade interpessoal na atenção à saúde. Essa superutilização pode estar relacionada ao fenômeno da "porta giratória" 
Fatores associados à Continuidade Interpessoal na Atenção à Saúde (CIAS) na população de Pelotas, Rio Grande do Sul, Brasil, 2005 (N = 3.133).

\begin{tabular}{|c|c|c|c|c|c|c|c|}
\hline Variáveis & $\begin{array}{l}\text { Prevalência } \\
\text { de CIAS (\%) }\end{array}$ & $\begin{array}{l}\text { Análise } \\
\text { bruta }\end{array}$ & IC95\% & Valor $\mathrm{p}$ & $\begin{array}{l}\text { Análise } \\
\text { ajustada }\end{array}$ & IC95\% & Valor p \\
\hline Sexo & & & & $<0,001$ & & & $<0,001$ * \\
\hline Masculino & 31,6 & 1,00 & & & 1,00 & & \\
\hline Feminino & 53,2 & 1,68 & $1,54-1,84$ & & 1,66 & $1,52-1,80$ & \\
\hline Idade (anos) & & & & $<0,001$ & & & $<0,001 * \star$ \\
\hline $20-29$ & 31,9 & 1,00 & & & 1,00 & & \\
\hline $30-39$ & 38,2 & 1,20 & $1,04-1,38$ & & 1,19 & $1,03-1,37$ & \\
\hline $40-49$ & 43,3 & 1,36 & $1,18-1,55$ & & 1,30 & $1,14-1,48$ & \\
\hline $50-59$ & 49,4 & 1,55 & $1,35-1,78$ & & 1,47 & $1,29-1,67$ & \\
\hline $60 \mathrm{ou}+$ & 59,7 & 1,87 & $1,65-2,12$ & & 1,76 & $1,56-1,99$ & \\
\hline Renda familiar (salários mínimos) & & & & $<0,001$ & & & $<0,001$ ** \\
\hline$<1$ & 35,5 & 1,00 & & & 1,00 & & \\
\hline $1-3$ & 35,3 & 0,99 & $0,85-1,17$ & & 1,02 & $0,87-1,20$ & \\
\hline $3-6$ & 47,4 & 1,33 & $1,14-1,56$ & & 1,35 & $1,16-1,58$ & \\
\hline 6 ou + & 56,2 & 1,58 & $1,36-1,84$ & & 1,61 & $1,39-1,87$ & \\
\hline Consulta no último ano & & & & $<0,001$ & & & $<0,001 *$ \\
\hline Não & 26,1 & 1,00 & & & 1,00 & & \\
\hline Sim & 50,2 & 1,92 & $1,70-2,17$ & & 1,47 & $1,29-1,67$ & \\
\hline Doença crônica & & & & $<0,001$ & & & 0,02 * \\
\hline Não & 39,2 & 1,00 & & & 1,00 & & \\
\hline Sim & 54,3 & 1,38 & $1,28-1,50$ & & 1,10 & $1,01-1,19$ & \\
\hline Autopercepção de saúde & & & & 0,01 & & & 0,8 ** \\
\hline Excelente & 39,8 & 1,00 & & & 1,00 & & \\
\hline Muito boa & 44,3 & 1,11 & $0,95-1,31$ & & 0,97 & $0,84-1,13$ & \\
\hline Boa & 42,8 & 1,08 & $0,94-1,23$ & & 1,00 & $0,89-1,14$ & \\
\hline Regular & 45,3 & 1,14 & $0,99-1,31$ & & 0,99 & $0,86-1,15$ & \\
\hline Ruim & 53,9 & 1,35 & $1,12-1,63$ & & 1,10 & $0,90-1,34$ & \\
\hline Forma do financiamento da consulta & & & & $<0,001$ & & & $<0,001$ * \\
\hline Pública & 31,6 & 1,00 & & & 1,00 & & \\
\hline Convênio & 54,2 & 1,72 & $1,57-1,88$ & & 1,37 & $1,24-1,52$ & \\
\hline Particular & 59,2 & 1,88 & $1,64-2,14$ & & 1,64 & $1,42-1,90$ & \\
\hline
\end{tabular}

* Teste de Wald de heterogeneidade;

** Teste de Wald para tendência linear.

em que os indivíduos realizam muitas consultas em vários locais. Esse fenômeno, bastante estudado em reinternações psiquiátricas, 19,20 mostra-se associado a diferenças sócio-econômicas, fatores culturais e baixa resolubilidade do cuidado médico. A "porta giratória" pode aumentar o número de consultas sem que os "superutilizadores” vinculem-se a algum serviço de saúde.

O sistema público local, organizado em múltiplas portas de entrada descentralizadas, repercute na facilidade de acesso, mas estas parecem não estar relacionadas a maiores prevalências de continuidade interpessoal na atenção à saúde; estes evidentes esforços de democratização do acesso à saúde foram acompanhados apenas recentemente de tentativas de vinculação da população às equipes de saúde, com o advento do PSF e da adscrição da população. Mas a adscrição pode ter efeito paradoxal, pois a vinculação também pode prejudicar a continuidade interpessoal na atenção à saúde ao retirar a autonomia dos indivíduos em consultar em outros locais com profissionais que gostariam de vincular-se.

O estudo brasileiro prévio encontrou 37,4\% de continuidade interpessoal na atenção à saúde, uma prevalência mais baixa que neste estudo. Porém, o estudo anterior incluiu populações mais jovens, que apresentam menores prevalências de 
Fatores associados à Continuidade Interpessoal na Atenção à Saúde (CIAS) em 1.052 indivíduos que consultam unidades básicas de saúde. Pelotas, Rio Grande do Sul, Brasil.

\begin{tabular}{|c|c|c|c|c|c|c|c|}
\hline Variáveis & $\begin{array}{l}\text { Prevalência } \\
\text { de CIAS (\%) }\end{array}$ & $\begin{array}{c}\text { Análise } \\
\text { bruta }\end{array}$ & IC95\% & Valor $\mathrm{p}$ & $\begin{array}{l}\text { Análise } \\
\text { ajustada }\end{array}$ & IC95\% & Valor $p$ \\
\hline Sexo & & & & $<0,001$ & & & $<0,001$ * \\
\hline Masculino & 21,4 & 1,00 & & & 1,00 & & \\
\hline Feminino & 43,0 & 2,00 & $1,63-2,46$ & & 1,97 & $1,61-2,42$ & \\
\hline Idade (anos) & & & & $<0,001$ & & & $<0,001 * \star$ \\
\hline $20-29$ & 24,0 & 1,00 & & & 1,00 & & \\
\hline $30-39$ & 37,2 & 1,55 & $1,19-2.03$ & & 1,52 & $1,19-2,01$ & \\
\hline $40-49$ & 32,7 & 1,36 & $1,03-1,80$ & & 1,31 & $1,00-1,74$ & \\
\hline $50-59$ & 38,9 & 1,62 & $1,21-2,18$ & & 1,56 & $1,17-2,08$ & \\
\hline $60 \mathrm{ou}+$ & 49,0 & 2,04 & $1,56-2,67$ & & 1,97 & $1,52-2,57$ & \\
\hline Consulta no último ano & & & & $<0,001$ & & & 0,01 * \\
\hline Não & 24,9 & 1,00 & & & 1,00 & & \\
\hline Sim & 39,1 & 1,57 & $1,28-1,94$ & & 1,31 & $1,06-1,62$ & \\
\hline Programa Saúde da Família & & & & 0,03 & & & 0,04 * \\
\hline Não & 31,8 & 1,00 & & & 1,00 & & \\
\hline Sim & 38,6 & 1,21 & $1,02-1,43$ & & 1,19 & $1,01-1,40$ & \\
\hline
\end{tabular}

* Teste de Wald de heterogeneidade;

** Teste de Wald para tendência linear.

continuidade interpessoal na atenção à saúde 14 . A literatura internacional relata prevalências superiores, mas pela diversidade de indicadores há limitações quanto à comparabilidade.

Os fatores associados à continuidade interpessoal na atenção à saúde na população foram: sexo feminino, aumento da idade, maior renda familiar, ter consultado no último ano, financiamento não-público da consulta e relato de doença crônica. Entre aqueles que consultam em unidades básicas de saúde, os fatores associados à continuidade interpessoal na atenção à saúde foram o sexo feminino, aumento da idade, ter consultado no ultimo ano e consultar em PSF.

Nesta pesquisa, os cuidados com a amostragem e a baixa taxa de perdas e recusas permitiram que este estudo fosse representativo da população de 20 anos ou mais, residente na zona urbana da cidade. O desfecho foi operacionalizado à semelhança de outro estudo brasileiro, a fim de tornar-se comparável 14. Entretanto, essa definição pode ter sido afetada por um viés de memória, pois a lembrança do nome do médico poderia ser maior entre os jovens quando comparados com os mais velhos. Esse viés teria efeito em direção à unidade.

O tema também se situa no centro de uma discussão acerca da conceituação de continui- dade e longitudinalidade, em que múltiplos fenômenos usam uma mesma terminologia. Neste estudo optou-se por um indicador que mede a continuidadeinterpessoalnaatençãoà saúde.Para isso, procurou-se captar um indicador de confiança em um determinado profissional, caracterizado pela lembrança do nome do médico 1,14 e pelo longo vínculo com este profissional, mensurado pela exigência de consultar com este médico há mais de um ano. A limitação desse indicador é que esse não inclui nenhuma medida de utilização entre seus componentes. O indivíduo pode identificar seu médico pelo nome mesmo que não tenha consultado há muito tempo, no entanto, ao incluirmos na análise somente aqueles que consultaram no último ano, os efeitos sobre a continuidade interpessoal na atenção à saúde foram semelhantes.

Como era esperado, a continuidade interpessoal na atenção à saúde esteve associada ao sexo feminino, resultado consistente com o estudo brasileiro 14. Estudos prévios justificam esse achado em função das mulheres consultarem mais, cuidarem mais de sua saúde, relatarem mais problemas de saúde 21 e possuírem maiores necessidades de consultas preventivas, como pré-natal e prevenção de câncer ginecológico. Esses padrões de cuidados em saúde femininos 
permitem uma maior proximidade das mulheres com o profissional médico, estabelecendo-se melhores níveis de confiança e maiores possibilidades de continuidade interpessoal na atenção à saúde.

$\mathrm{O}$ aumento da idade esteve associado linearmente com a continuidade interpessoal na atenção à saúde, fato já identificado por estudos anteriores 14,15. Esse achado deve-se não apenas ao aparecimento de morbidades e maiores preocupações com a saúde 22, mas também ao avançar da idade que impõe intervenções preventivas, curativas e de reabilitação. Já os jovens usualmente procuram serviços de saúde para tratamento de doenças, privilegiando atendimentos de fácil acesso.

A renda familiar também se mostrou diretamente associada à continuidade interpessoal na atenção à saúde, pois aqueles que possuem maiores rendas se vinculam a serviços que oferecem maiores facilidades de acesso e possibilidades de continuidade interpessoal na atenção à saúde 23 . Indivíduos com menores rendas são mais suscetíveis à perda da continuidade interpessoal na atenção à saúde com variantes do sistema, como a mudança do local de atendimento ou mesmo o descredenciamento de convênios. Maiores rendas permitem que o indivíduo "opte" por modelos assistenciais que permitam consultar com quem desejam ou ainda pagar diretamente para ter continuidade interpessoal na atenção à saúde. Esse resultado é consistente com outros estudos, inclusive levando-se em conta outros indicadores de condição sócio-econômica como classe social 14 e escolaridade.

A prevalência de consultas no último ano foi alta $(73,2 \%)$, mas coincidente com estudos prévios que mostram uma superutilização de consultas médicas em Pelotas 17. No Brasil, em 2003, $62,8 \%$ de todos os brasileiros consultaram um médico 21 . A associação entre continuidade interpessoal na atenção à saúde e consultar no último ano provavelmente indica que quem mais utiliza serviços de saúde tem mais chances de vincular-se a um determinando profissional, e também relata maior preocupação com a saúde. Contudo, essa associação pode estar afetada por causalidade reversa, uma vez que continuidade interpessoal na atenção à saúde pode ser preditor de utilização de serviços de saúde.

A forma de financiamento das consultas mostra um predomínio das consultas pelo SUS e por planos de saúde, em detrimento das consultas privadas. Em 2003, a Pesquisa Nacional por Amostra de Domicílios (PNAD 2003) mostrou que $24,6 \%$ da população estavam cobertas por planos de saúde. Esses porcentuais mostram, ao mesmo tempo, uma migração da população para planos de saúde e uma diminuição importante da remuneração privada 21 . A associação entre continuidade interpessoal na atenção à saúde e formas de pagamentos privadas e conveniadas, quando controlada para renda, mostra que os planos de saúde, em geral, oferecem maiores possibilidades de vínculo entre o médico e seus pacientes do que o sistema público.

Em geral, tanto os convênios como os atendimentos particulares oferecem a possibilidade de consultar e, quando necessário, internar com o mesmo médico, enquanto no sistema público esta possibilidade é remota. O sistema público inglês vincula seus pacientes com um determinado médico, mediante contratualização pessoal entre o médico e seu paciente 24,25 . O sistema brasileiro estimula vínculos com os serviços de saúde, entretanto estudos prévios mostram a clara superioridade do vínculo com o médico quando comparado ao vínculo com um local, para consultas preventivas 8,14 , acesso às consultas 26 e menor ocorrência da hospitalização ${ }^{3}$. A contratualização inglesa poderia justificar as altas prevalências de continuidade interpessoal na atenção à saúde em estudo de base populacional na Inglaterra 15 .

A prevalência de doença crônica de $30 \%$ foi semelhante à encontrada pela PNAD 2003 $(29,9 \%)$ e também esteve associada à continuidade interpessoal na atenção à saúde, em função de uma maior valorização do vínculo com um médico cuidador por populações vulneráveis 27 , já evidenciada em uma revisão prévia ${ }^{28}$. A maior necessidade de cuidados com a doença, como por exemplo, fornecimento de receitas, mudanças no tratamento e realização de exames de rotina também justificariam tais achados. Essa associação pode também estar afetada pela causalidade reversa, pois aqueles que se vinculam a seus médicos têm maiores possibilidades diagnósticas.

Este estudo não encontrou associação significativa entre autopercepção ruim sobre a saúde e continuidade interpessoal na atenção à saúde. Esse achado não é consistente com MendozaSassi \& Beria ${ }^{14}$, em 2003, porém nesse estudo o recordatório de percepção de saúde incluiu apenas no último ano. Esse achado poderia indicar que a insatisfação com a saúde está relacionada à troca freqüente de médico.

A análise realizada apenas com aqueles que consultam em unidades básicas de saúde mostrou que as mulheres, o aumento da idade e consultar no último ano, assim como na avaliação de base populacional, tiveram mais continuidade interpessoal na atenção à saúde. A renda não esteve associada, provavelmente porque a população que consulta em unidades básicas de saúde 
seja muito semelhante em relação às condições sócio-econômicas.

Aqueles que consultam em PSF tiveram mais chances de ter continuidade interpessoal na atenção à saúde quando comparados com aqueles que consultam em unidades tradicionais. Este estudo não caracterizou se o médico de referência é o profissional do programa de saúde da família, porém, é provável que isto ocorra na maioria das vezes. Nesse caso, a capacidade de estabelecer vínculos entre o médico e seus pacientes pode ser um importante marcador de qualidade na atenção à saúde associado ao PSF. Os efeitos dessa associação podem estar afetados pela transferência de oito médicos do PSF de local, ocorrida em setembro de 2005, dois meses antes da coleta dos dados. Essa iniciativa, símbolo da rotatividade dos médicos nas equipes, pode ter provocado um viés em direção à unidade e mostra a desvalorização da continuidade interpessoal na atenção à saúde por parte do gestor público.

Os resultados deste estudo mostram que a prevalência de continuidade interpessoal na atenção à saúde é baixa quando comparada ao sistema privado de saúde, embora o sistema público tenha uma proposta de integralidade que inclua ações contínuas de saúde. Com a implantação do PSF, surgem novos conceitos, como a responsabilização e adscrição da equipe de saúde sobre uma parcela da população. Esses instrumentos parecem estar promovendo continuidade interpessoal na atenção à saúde, embora com resultados ainda inferiores à prática privada. Alguns estudos sobre o PSF em cidades médias e grandes têm mostrado dificuldades em sua implantação 12 , enquanto outros, apontam que o PSF tem sido bem sucedido na promoção da eqüidade 29 . Os achados de nosso estudo concordam com este último, e mostram que a responsabilização e vinculação dos pacientes estão ocorrendo.

A continuidade interpessoal na atenção à saúde é mais prevalente em alguns grupos populacionais que necessitam maior vínculo com seus médicos, como os idosos e aqueles com doenças crônicas. No entanto, outros grupos vulneráveis, como aqueles de baixa renda e usuários do sistema público de saúde, tiveram menores prevalências, o que mostra importante iniqüidade em saúde. Este estudo mostrou que o PSF está cumprindo um importante papel na responsabilização e vinculação dos pacientes a seus médicos, mas ainda há um longo caminho a ser percorrido, mediante políticas que reduzam a rotatividade de profissionais e melhorem o acolhimento nas unidades básicas de saúde.

O sistema público brasileiro precisa estimular a continuidade interpessoal na atenção à saúde, mediante políticas públicas capazes de reduzir a desigualdade no acesso á saúde de qualidade, estimulando os pacientes a acessar o sistema por uma atenção primária e pelo serviço responsável pelo cuidado, a fim de garantir o vínculo e a responsabilização, principalmente em grupos populacionais menos privilegiados economicamente.

Embora a continuidade tenha sido objeto de pesquisas nas últimas décadas, nos últimos anos houve um esforço para redefinir e precisar sua conceituação e operacionalização. Futuros estudos que utilizem uma definição padronizada permitirão avaliar melhor a consistência dos achados acerca da continuidade interpessoal na atenção à saúde. É necessário também que futuros estudos ampliem a concepção de continuidade interpessoal na atenção à saúde, comparando os diferentes tipos de continuidade, de forma a avaliar se o modelo brasileiro que tem ênfase em uma equipe ampla tem desempenho adequado quando comparado ao vínculo apenas com o médico.

É necessário aprofundar o conhecimento sobre os determinantes da continuidade interpessoal na atenção à saúde, detalhando as características pessoais e profissionais dos médicos que são fundamentais no estabelecimento de responsabilização e vínculo com seus pacientes. No Brasil, há ainda a necessidade de estudos posteriores sobre o impacto da continuidade interpessoal na atenção à saúde sobre o processo saúde/doença, os hábitos de vida e o uso dos serviços de saúde. 


\section{Resumo}

Estudar a prevalência de continuidade interpessoal na atenção à saúde e seus determinantes. Foi realizado um estudo transversal de base populacional com 3.133 indivíduos com 20 anos ou mais da zona urbana de Pelotas, Rio Grande do Sul, Brasil. A análise multivariável foi realizada através de regressão de Poisson, tendo no primeiro nível variáveis sócio-econômicas e demográficas e no nível proximal, variáveis assistenciais e de necessidades em saúde. A prevalência de continuidade interpessoal na atenção à saúde foi de $43,7 \%$ (IC95\%: 42,0-45,5). Sexo feminino, idades avançadas, maior renda, consulta no último ano, relato de doença crônica e não consultarem no sistema público de saúde apresentaram maior continuidade interpessoal na atenção à saúde. Entre os que consultam em unidades básicas de saúde, sexo feminino, aumento da idade e o Programa Saúde da Família estiveram associadas com continuidade interpessoal na atenção à saúde. Alguns grupos vulneráveis, como aqueles de baixa renda e usuários do sistema público de saúde, apresentaram menores prevalências do desfecho.

Continuidade da Assistência ao Paciente; Atenção Primária à Saúde; Relações Médico-Paciente

\section{Referências}

1. Saultz JW. Defining and measuring interpersonal continuity of care. Ann Fam Med 2003; 1:134-43.

2. Hjortdahl P. Ideology and reality of continuity of care. Fam Med 1990; 22:361-4.

3. Mainous $3^{\text {rd }}$ AG, Gill JM. The importance of continuity of care in the likelihood of future hospitalization: is site of care equivalent to a primary clinician? Am J Public Health 1998; 88:1539-41.

4. Wasson JH, Sauvigne AE, Mogielnicki RP, Frey WG, Sox CH, Gaudette C, et al. Continuity of outpatient medical care in elderly men. A randomized trial. JAMA 1984; 252:2413-7.

5. Gill JM, Mainous $3^{\text {rd }}$ AG. The role of provider continuity in preventing hospitalizations. Arch Fam Med 1998; 7:352-7.

\section{Colaboradores}

A. G. Fassa eV. M. V. Paniz orientaram a pesquisa, a análise dos dados e a redação do artigo. L. A. Rosa Filho fez a análise dos dados, revisou a literatura e redigiu o artigo.
6. Christakis DA, Mell L, Koepsell TD, Zimmerman FJ, Connell FA. Association of lower continuity of care with greater risk of emergency department use and hospitalization in children. Pediatrics 2001; 107:524-9.

7. Saultz JW, Lochner J. Interpersonal continuity of care and care outcomes: a critical review. Ann Fam Med 2005; 3:159-66.

8. Ettner SL. The relationship between continuity of care and the health behaviors of patients: does having a usual physician make a difference? Med Care 1999; 37:547-55.

9. Piccini RX, Victora CG. How well is hypertension managed in the community? A population-based survey in a Brazilian city. Cad Saúde Pública 1997; 13:595-600. 
10. Parchman ML, Pugh JA, Noel PH, Larme AC. Continuity of care, self-management behaviors, and glucose control in patients with type 2 diabetes. Med Care 2002; 40:137-44.

11. Brasil. Portaria no. 648, de 28 de março de 2006. Diário Oficial da União 2006; 29 mar.

12. Escorel S, Mendonça MH, Magalhães R, Senna MCM. Saúde da família: avaliação da implementação em dez grandes centros urbanos. Síntese dos principais resultados. Brasília: Ministério da Saúde; 2005.

13. Haggerty JL, Reid RJ, Freeman GK, Starfield BH, Adair CE, McKendry R. Continuity of care: a multidisciplinary review. BMJ 2003; 327:1219-21.

14. Mendoza-Sassi R, Beria JU. Prevalence of having a regular doctor, associated factors, and the effect on health services utilization: a population-based study in Southern Brazil. Cad Saúde Pública 2003; 19:1257-66.

15. Doescher MP, Saver BG, Fiscella K, Franks P. Preventive care. J Gen Intern Med 2004; 19:632-7.

16. Associação Brasileira de Empresas de Pesquisa. Critério de classificação econômica do Brasil. São Paulo: Associação Nacional Brasileira de Pesquisa; 2002.

17. Costa JSD, Facchini LA. Utilização de serviços ambulatoriais em Pelotas: onde a população consulta e com que freqüência. Rev Saúde Pública 1997; 31:360-9.

18. Capilheira MF, Santos IS. Fatores individuais associados à utilização de consultas médicas por adultos. Rev Saúde Pública 2006; 40:436-43.

19. Gastal FL, Andreoli SB, Quintana MI, Almeida Gameiro M, Leite SO, McGrath J. Predicting the revolving door phenomenon among patients with schizophrenic, affective disorders and non-organic psychoses. Rev Saúde Pública 2000; 34:280-5.
20. Surber RW, Winkler EL, Monteleone M, Havassy BE, Goldfinger SM, Hopkin JT. Characteristics of high users of acute psychiatric inpatient services. Hosp Community Psychiatry 1987; 38:1112-4.

21. Instituto Brasileiro de Geografia e Estatística. Pesquisa Nacional por Amostra de Domicílios. Rio de Janeiro: Instituto Brasileiro de Geografia e Estatística; 2003.

22. Menec VH, Roos NP, Black C, Bogdanovic B. Characteristics of patients with a regular source of care. Can J Public Health 2001; 92:299-303.

23. Menec VH, Sirski M, Attawar D. Does continuity of care matter in a universally insured population? Health Serv Res 2005; 40:389-400.

27. Guthrie B. Continuity in UK general practice: a multilevel model of patient, doctor and practice factors associated with patients seeing their usual doctor. Fam Pract 2002; 19:496-9.

28. Mainous 3rd AG, Baker R, Love MM, Gray DP, Gill JM. Continuity of care and trust in one's physician: evidence from primary care in the United States and the United Kingdom. Fam Med 2001; 33:22-7.

29. Lambrew JM, DeFriese GH, Carey TS, Ricketts TC, Biddle AK. The effects of having a regular doctor on access to primary care. Med Care 1996; 34:138-51.

30. Nutting PA, Goodwin MA, Flocke SA, Zyzanski SJ, Stange KC. Continuity of primary care: to whom does it matter and when? Ann Fam Med 2003; 1:149-55.

31. Pandhi N, Saultz JW. Patients' perceptions of interpersonal continuity of care. J Am Board Fam Med 2006; 19:390-7.

32. Facchini LP, Piccini RX, Tomasi E, Thumé E, Silveira DS, Siqueira FV, et al. Desempenho do PSF no Sul e no Nordeste do Brasil: avaliação institucional e epidemiológica da atenção básica à saúde. Ciênc Saúde Coletiva 2006; 11:669-81.

Recebido em 04/Dez/2006

Versão final reapresentada em 31/Jul/2007

Aprovado em 05/Set/2007 